\title{
How many cores should be taken in a repeat biopsy on patients in whom atypical small acinar proliferation has been identified in an initial transrectal prostate biopsy?
}

Erdogan Aglamis ${ }^{1}$, Ramazan Kocaarslan², Ugur Yucetas ${ }^{3}$, Gokhan Toktas³, Cavit Ceylan4, Omer Gokhan Doluoglu ${ }^{5}$, Erdinc Unluer ${ }^{2}$

${ }^{1}$ Clinics of Urology, Elazig Education and Research Hospital, Elazig; ${ }^{2}$ Department of Urology, Kafkas University Medical Faculty Kars; ${ }^{3}$ Clinics of Urology, Istanbul Education and Research Hospital, Istanbul; ${ }^{4}$ Clinics of Urology, Yuksek Ihtisas Education and Research Hospital and ${ }^{5}$ Clinics of Urology, Ankara Education and Research Hospital, Ankara, Turkey

\section{ABSTRACT}

Objective: To compare cancer detection rates according to the number of biopsy cores in patients on whom a repeat prostate biopsy was performed for atypical small acinar proliferation (ASAP).

Materials and Methods: The data of 4950 consecutive patients on whom prostate biopsies were performed were assessed retrospectively. A total of 107 patients were identified as having ASAP following an initial prostate biopsy, and they were included in the study. A six-core prostate biopsy (PBx) was performed on 15 of the 107 patients, $12 \mathrm{PBx}$ on 32 patients, and $20 \mathrm{PBx}$ on 60 patients. Cancer detection rates were compared according to the number of biopsy cores. The localization of the cancer foci was also evaluated.

Results: The cancer detection rates in patients on whom $6 \mathrm{PBx}, 12 \mathrm{PBx}$, and $20 \mathrm{PBx}$ were performed were 20\% (3/15), 31\% (10/32), and 58\% (35/60), respectively, and a statistically significant difference was found $(\mathrm{p}=0.005)$. When cancer detection rates in patients with total prostate specific antigen (PSA) $<10 \mathrm{ng} / \mathrm{mL}$, PSA density $\geq 0.15$, normal digital rectal examination, and prostate volume $\geq 55 \mathrm{~mL}$ were compared according to the number of biopsy cores, a significant difference was identified ( $p=0.02,0.03,0.006$, and 0.04, respectively). Seventy-five percent of the foci where cancer was detected were at the same and/or adjacent sites as the ASAP foci in the initial biopsy, and 54\% were identified in contralateral biopsies in which ASAP foci were present.

Conclusion: As the biopsy core number increases, the cancer detection rate increases significantly in patients on whom a repeat biopsy is performed due to ASAP. The highest cancer rate is found in 20-core repeat biopsies performed equally from all foci.

\section{ARTICLE INFO}

Key words:

Prostatic Intraepithelial Neoplasia; Biopsy; Prostatic

Neoplasms; Ultrasound, High-Intensity Focused,

Transrectal

Int Braz J Urol. 2014; 40: 605-12

Submitted for publication: October 10, 2013

Accepted after revision: January 22, 2014

\section{INTRODUCTION}

Prostate cancer is one of the most common cancer in men (1). Prostate biopsy is the standard method used to diagnose prostate cancer. Biopsy procedures in organs with cancers are usually car- ried out for the histopathological assessment of tumors diagnosed clinically. This is also done to confirm tumor suspicion in other instances, such as T1 prostate cancer. While positive biopsy results can verify a prostate cancer diagnosis, negative biopsies may cause the persistence of cancer suspicion (2). 
High serum total prostate specific antigen (PSA) and/or prostate cancer suspicion in digital rectal examinations require prostate biopsies. In patients with initial negative biopsy results, cancer detection rates with a repeat biopsy vary between 10 and 25\% (3,4). Histopathological findings identified in the initial biopsy, in addition to findings of PSA parameters and digital rectal examination (DRE), also are important in deciding whether or not to perform a repeat biopsy.

In patients whose initial prostate biopsy indicated a negative result, histopathological findings, such as benign prostatic hyperplasia, conditions including prostatic intraepithelial neoplasia (PIN), atypical small acinar proliferation (ASAP), and prostatic inflammation, have also been reported. ASAP is present in 1.5-24\% of needle biopsies $(5,6)$. However, pathologists do not have adequate data regarding the potential for cancer in ASAP with the definition of "focal foci arising from small acinar structures constituted by atypical epithelium cells" and are therefore undecided in terms of cancer diagnoses. Therefore, repeat biopsies should be performed in these cases. The cancer detection rates in repeat biopsies of these cases vary between $19-38 \%(7,8)$. The core number of the repeat biopsy and the area in which it should be performed has frequently been the subject of research.

Nowadays, repeat prostate biopsies are commonly carried out. In this study, we compared cancer detection rates in relation to the biopsy core number in patients who had had a repeat prostate biopsy if ASAP was reported in the initial transrectal prostate biopsy. We assessed the localization of foci where cancer was detected as well as the cancer detection rates according to the PSA parameters and clinical findings in the repeat biopsy.

\section{MATERIALS AND METHODS}

The data of 4950 consecutive patients on whom a prostate needle biopsy was performed under the guidance of transrectal ultrasonography between January 1998 and October 2012 were assessed retrospectively. Prostate biopsies (PBx) under the guidance of transrectal ultrasonography were carried out on patients with abnormal findings following digital rectal examinations (DRE) and/or prostate cancer suspicion due to high serum total PSA (tPSA). Technique and machinery has changed throughout these years. Therefore the biopsy groups might be heterogeneous. Antibiotic treatment was started two days before the biopsy procedure and continued for three days after the procedure. A rectal enema was applied 1-2 hours before the biopsy procedure. ASAP without any findings of malignancy was noted in 5.1\% of the results of the initial prostate biopsies. ASAP is described as the presence of atypical glands not diagnostic of prostate cancer, and/or the absence of definitive architectural or cytological features of carcinoma $(7,9)$. A repeat biopsy was performed on patients in whom ASAP was identified in the initial biopsy and who accepted a repeat biopsy. The repeat biopsy was performed six months after the initial biopsy.

As 147 (58\%) of the 254 patients on whom an initial prostate biopsy was performed and ASAP was identified either did not go for a follow-up or refused a further biopsy procedure, it was not possible to do repeat biopsies on these patients. Repeat biopsies were performed on 107 (42\%) of the 254 patients in whom ASAP had been identified in the initial biopsy. These 107 patients were therefore included in our study. While repeat biopsies were performed on $107 \mathrm{pa}$ tients, 6-core prostate biopsies (6 PBx) were carried out on $15(14 \%)$ patients, 12-core prostate biopsies (12 PBx) on 32 (30\%) patients, and 20core prostate biopsies (20 $\mathrm{PBx})$ in $60(56 \%)$ patients. Prostate volume was calculated using the formula for a prostate ellipsoid, i.e., transverse length $\mathrm{x}$ transverse width $\mathrm{x}$ longitudinal height $x$ 0.52. PSA density (PSAD) was calculated by dividing tPSA by prostate volume.

The histopathological results of all 107 patients on whom a 6-core, 12-core, and 20-core repeat biopsy were performed were assessed. The cancer detection rates in the patients were compared according to the repeat biopsy core number. The cancer detection rates in the repeat biopsies were also compared according to the findings of tPSA $(<10$ and $\geq 10 \mathrm{ng} / \mathrm{mL})$, PSAD $(<0.15$ and $\geq$ $0.15)$, prostate volume $(<55$ and $\geq 55 \mathrm{~mL}$ ), and 
DRE (normal and abnormal). The localization of foci where cancer was detected in the repeat biopsies was evaluated according to ASAP foci.

\section{Statistical analysis}

Statistical analyses was performed using SPSS $^{\circledR} 16.0$ software. The data were presented as mean \pm standard deviation. A one-way ANOVA test was used to compare the ages, prostatic volumes, PSA densities, and serum tPSA levels of the patients according to the number of biopsy cores performed. The chi-square test was used to analyze the differences in cancer detection rates for the 6-PBx, 12-PBx, and 20-PBx groups. Furthermore, the cancer detection rates based on IPSA, PSAD, prostate volume, and digital rectal examination status were analyzed according to the 6-PBx, 12-PBx, and 20$\mathrm{PBx}$ groups. $\mathrm{P}$ values $<0.05$ were considered statistically significant.

\section{RESULTS}

The mean age, tPSA, PSAD, and prostate volume values of the 107 patients included in the study were $63.33 \pm 7.00,8.01 \pm 4.68,0.18 \pm 0.15$, and $48.31 \pm 22.15$, respectively. No significant difference was determined between age, tPSA, PSAD, and prostate volumes of the patients on whom a 6-core, 12-core, or 20-core repeat biopsy was per- formed ( $\mathrm{P}$ values 0.305, 0,684, 0.455, and 0.347, respectively) (Table-1).

Prostate cancer was identified in 20\% (3/15) of the patients with a 6-core repeat biopsy, $31 \%(10 / 32)$ of the patients with a 12-core repeat biopsy, and 58\% (35/60) of the patients with a 20-core repeat biopsy (Table-2). A statistically significant increase was thus determined in the cancer detection rates concomitant to an increase in the core number of the repeat biopsy $(p=0.005)$. When the cancer detection rates were compared separately for the 6-PBx, 12-PBx, and 20-PBx groups according to the findings of serum tPSA, PSAD, prostate volume and DRE, no significant differences were identified (Table-2). On the other hand, the cancer detection rates were compared between the 6-PBx, 12-PBx, and 20-PBx groups according to the serum $\mathrm{PSA}$, PSAD, prostate volume, and DRE results, and statistically significant differences were found in the cancer detection rates of the patients with $\mathrm{PSA}<10 \mathrm{ng} / \mathrm{mL}, \mathrm{PSAD} \geq$ 0.15 , prostate volume $\geq 55 \mathrm{~mL}$, and normal DRE ( $\mathrm{P}$ values $0.024,0.039,0.044$, and 0.006 , respectively) (Table-2). The cancer detection rate in the patients who had had $20 \mathrm{PBx}$ was significantly higher (Table-2).

In the histopathological evaluation of the 107 patients on whom repeat biopsies were performed because of ASAP, prostate cancer was present in $45 \%(48 / 107)$ of cases, ASAP in $12 \%$

Table 1 - Patient characteristics.

\begin{tabular}{lcccc}
\hline Variable & $6 \mathrm{PBx}$ & $12 \mathrm{PBx}$ & $20 \mathrm{PBx}$ & $\mathrm{p}^{*}$ \\
\hline No.of patients & 15 & 32 & 60 & \\
Age Mean \pm SD & $64.80 \pm 4.16$ & $64.37 \pm 7.06$ & $62.41 \pm 7.47$ & 0.305 \\
tPSA (ng/mL) Mean \pm SD & $8.71 \pm 3.04$ & $8.31 \pm 5.20$ & $7.68 \pm 4.75$ & 0.684 \\
PSA Density Mean \pm SD & $0.22 \pm 0.10$ & $0.20 \pm 0.24$ & $0.17 \pm 0.08$ & 0.455 \\
PV (mL) Mean \pm SD & $44.06 \pm 16.84$ & $52.87 \pm 25.96$ & $46.95 \pm 21.03$ & 0.347 \\
No. Normal DRE (\%) & $12 / 15(80 \%)$ & $23 / 32(72 \%)$ & $52 / 60(87 \%)$ & \\
No. Abnormal DRE $(\%)$ & $3 / 15(20 \%)$ & $9 / 32(28 \%)$ & $8 / 60(13 \%)$ & \\
\hline
\end{tabular}

*One-Way ANOVA

PBx = Prostate biopsy; $\mathbf{P S A}=$ Prostate specific antigen; $\mathbf{P V}=$ Prostate volume; $\mathbf{D R E}=$ Digital rectal examination 
Table 2 - Cancer detection rates according to different parameters.

\begin{tabular}{|c|c|c|c|c|c|}
\hline Variable & & $\begin{array}{c}6 \mathrm{PBx} \\
\text { No/Total No Ca }\end{array}$ & $\begin{array}{c}12 \mathrm{PBx} \\
\text { No/Tota No Ca }\end{array}$ & $\begin{array}{c}20 \mathrm{PBx} \\
\text { No/Total No Ca }\end{array}$ & $p^{*}$ Value \\
\hline Overall & & $3 / 15(20 \%)$ & $10 / 32(31 \%)$ & $35 / 60(58 \%)$ & 0.005 \\
\hline \multicolumn{6}{|c|}{ tPSA (ng/mL) } \\
\hline & Less than 10 & $2 / 9(22 \%)$ & $8 / 26(31 \%)$ & $28 / 48(58 \%)$ & 0.024 \\
\hline & 10 or greater & $1 / 6(17 \%)$ & $2 / 6(33 \%)$ & $7 / 12(58 \%)$ & 0.214 \\
\hline & $P$ value & 0.792 & 0.393 & 1.0 & \\
\hline \multicolumn{6}{|c|}{ PSA Density } \\
\hline & Less than 0.15 & $1 / 5(20 \%)$ & $6 / 20(30 \%)$ & $17 / 31(55 \%)$ & 0.12 \\
\hline & 0.15 or greater & $2 / 10(20 \%)$ & $4 / 12(33 \%)$ & $18 / 29(62 \%)$ & 0.039 \\
\hline & $P$ value & 0.171 & 0.555 & 0.570 & \\
\hline \multicolumn{6}{|l|}{ PV (mL) } \\
\hline & Less than $55 \mathrm{~mL}$ & $2 / 9(22 \%)$ & $8 / 23(35 \%)$ & $22 / 39(56 \%)$ & 0.086 \\
\hline & $55 \mathrm{~mL}$ or greater & $1 / 6(17 \%)$ & $2 / 9(22 \%)$ & $13 / 21(62 \%)$ & 0.044 \\
\hline & $P$ value & 0.792 & 0.491 & 0.681 & \\
\hline \multicolumn{6}{|l|}{ DRE } \\
\hline & Normal & $2 / 12(17 \%)$ & $7 / 23(30 \%)$ & $31 / 52(60 \%)$ & 0.006 \\
\hline & Abnormal & $1 / 3(33 \%)$ & $3 / 9(33 \%)$ & $4 / 8(50 \%)$ & 0.757 \\
\hline & $P$ value & 0.519 & 0.874 & 0.608 & \\
\hline
\end{tabular}

* The chi-square test

$\mathbf{P B x}=$ Prostate biopsy; $\mathbf{P S A}=$ Prostate specific antigen; $\mathbf{C a}=$ Prostate cancer $\mathbf{P V}=$ Prostate volume; $\mathbf{D R E}=$ Digital rectal examination

(13/107), high-grade prostatic intraepithelial neoplasia (HGPIN) in 4\% (4/107), and benign prostate hyperplasia in 39\% (42/107) (Table-3).

The Gleason score was $\leq 5$ in one of the patients on whom a $6 \mathrm{PBx}$ was performed and prostate cancer was detected, and it was 6 in two such patients (Table-3). The Gleason score was $\leq$ 5 in three of the patients on whom a $12 \mathrm{PBx}$ was performed and prostate cancer was detected, 6 in six of the 12-PBx patients, and 7 in one of these patients (Table-3). The Gleason score was $\leq 5$ in eight patients on whom a $20 \mathrm{PBx}$ was performed and prostate cancer was detected, 6 in $2420-\mathrm{PBx}$ patients, and 7 in three such patients (Table-3).

When foci where cancer was detected in the repeat biopsies were in the same area as the
ASAP foci in the initial biopsy, cancer in the foci in contralateral biopsies was detected in 26 (54\%) of a total of 48 patients, cancer in the same foci as ASAP was in $8(17 \%)$ patients, and cancer in the ipsilateral adjacent foci was in 32 (67\%) patients (Table-4).

\section{DISCUSSION}

ASAP is frequently detected in prostate needle biopsies following abnormal DRE or increased tPSA (10). Previous studies have reported that prostate cancer was detected in $19-60 \%$ of cases where subsequent biopsies were performed following the diagnosis of ASAP; however, there was no discussion about patients receiving a repeat biopsy 
Table 3 - Pathological findings at repeat prostate biopsy for ASAP.

\begin{tabular}{|c|c|c|c|c|c|}
\hline Variable & & $\begin{array}{c}\text { Overall } \\
\text { No/Total No }\end{array}$ & $\begin{array}{c}6 \mathrm{PBx} \\
\text { No/Total No }\end{array}$ & $\begin{array}{c}12 \mathrm{~PB} \\
\text { No/Total No }\end{array}$ & $\begin{array}{c}20 \mathrm{PBx} \\
\text { No/Total No }\end{array}$ \\
\hline \multicolumn{6}{|c|}{ Repeat Biopsy Diagnosis } \\
\hline & $\mathrm{Ca}$ & $48 / 107(45 \%)$ & $3 / 15(20 \%)$ & $10 / 32(31 \%)$ & $35 / 60(58 \%)$ \\
\hline & ASAP & $13 / 107(12 \%)$ & $3 / 15(20 \%)$ & $5 / 32(16 \%)$ & $5 / 60(8 \%)$ \\
\hline & HGPIN & $4 / 107(4 \%)$ & & $3 / 32(9 \%)$ & $1 / 60(2 \%)$ \\
\hline & BPH & $42 / 107(39 \%)$ & $9 / 15(60 \%)$ & $14 / 32(44 \%)$ & $19 / 60(32 \%)$ \\
\hline \multicolumn{6}{|c|}{ Gleason Score } \\
\hline & $\leq 5$ & $12 / 48(25 \%)$ & $1 / 3(33 \%)$ & $3 / 10(30 \%)$ & $8 / 35(23 \%)$ \\
\hline & 6 & $32 / 48(67 \%)$ & $2 / 3(67 \%)$ & $6 / 10(60 \%)$ & $24 / 35(69 \%)$ \\
\hline & 7 & $4 / 48(8 \%)$ & & $1 / 10(10 \%)$ & $3 / 35(8 \%)$ \\
\hline
\end{tabular}

$\mathbf{C a}=$ Prostate cancer; ASAP = Atypical small acinar proliferation; $\mathbf{H G P I N}=$ High Grade prostatic intraepithelial neoplasia; $\mathbf{B P H}=$ Benign prostatic hyperplasia

Table 4 - Localization of foci where cancer was detected in repeat prostate biopsy (according to ASAP foci in the initial prostate biopsy).

\begin{tabular}{|c|c|c|c|c|}
\hline Variable & $\begin{array}{c}\text { Overall } \\
\text { No/TotalNo Ca }\end{array}$ & $\begin{array}{c}\text { 6PBx } \\
\text { No/Total No Ca }\end{array}$ & $\begin{array}{c}12 \mathrm{PBx} \\
\text { No/Total No Ca }\end{array}$ & $\begin{array}{c}20 \mathrm{PBx} \\
\text { No/Total No Ca }\end{array}$ \\
\hline In the same foci & $4 / 48(8 \%)$ & & $2 / 10(20 \%)$ & $2 / 35(6 \%)$ \\
\hline In the ipsilateral adjacent foci & $14 / 48(29 \%)$ & $2 / 3(67 \%)$ & $2 / 10(20 \%)$ & $10 / 35(28 \%)$ \\
\hline $\begin{array}{l}\text { In the same foci and ipsilateral adjacent } \\
\text { foci }\end{array}$ & $4 / 48(8 \%)$ & & & $4 / 35(11 \%)$ \\
\hline $\begin{array}{l}\text { In the ipsilateral adjacent foci and in the } \\
\text { contralateral foci }\end{array}$ & $14 / 48(29 \%)$ & & $4 / 10(40 \%)$ & $10 / 35(28 \%)$ \\
\hline In the contralateral foci & $12 / 48(25 \%)$ & $1 / 3(33 \%)$ & $2 / 10(20 \%)$ & $9 / 35(26 \%)$ \\
\hline
\end{tabular}

$\mathbf{C a}=$ Prostate cancer

in these cases. These studies suggest that a repeat biopsy, independent of PSA values, should be performed three to six months later in cases where ASAP is detected $(8,11,12)$. In our study, when the cancer detection rates were assessed separately in patients on whom a 6-core, 12-core, and 20core repeat biopsies were performed according to tPSA, PSAD, prostate volume, and DRE findings, no significant differences were found. Therefore, a decision to perform a repeat biopsy independently of PSA parameters and clinical findings would provide a more accurate outcome.
In a study by Ryu et al., ASAP was identified in $244(7.8 \%)$ of a total of 3130 patients on whom prostate biopsies were carried out, and a repeat biopsy was performed on 170 of them. Prostate cancer was detected in 57 (33.5\%) of these cases (13). Similarly to the literature, in our study ASAP was reported in 254 (5.1\%) of a total of 4950 patients. In $45 \%$ of the patients on whom a repeat biopsy was done due to ASAP, prostate cancer was identified. In our study, however, if a 20-core repeat biopsy had been performed on the patients on whom 6-core and 12-core repeat biopsies were 
carried out, the cancer detection rate would probably have been higher than 45\%. Because of the high cancer detection rates, a repeat biopsy should be performed in cases where ASAP is detected.

It has been reported that in ASAP cases where the cancer detection rate is high following a repeat biopsy, the regions where ASAP occurred in the initial biopsy should be overemphasized $(7,8)$. According to another study, cancer was detected in $84.8 \%$ of cases where a repeat biopsy was performed on the former biopsy area, and in 47.8\% of cases where ipsilateral adjacent and contralateral biopsies were performed. Accordingly, the performance of three biopsies on the former atypical area, three adjacent to the former area, three on the opposite side, and six on other sites were suggested (14). In our study, although cancer was detected in $17 \%$ of the former ASAP areas, it was detected in $67 \%$ of ipsilateral adjacent biopsies and 54\% of contralateral biopsies. In terms of a repeat biopsy, therefore, we consider that biopsies should be performed in equal numbers in all foci areas regardless of the former ASAP foci location. A high core number in the repeat biopsy on affected patients will increase the cancer detection rate and reduce the necessity for a second biopsy.

It has been reported that when prostate volume increases in patients in whom a six-core prostate biopsy has been carried out, the cancer detection rate decreases $(15,16)$. Similarly in our study, as long as the repeat biopsy core number increased in patients with a prostate volume $\geq 55 \mathrm{~mL}$, the cancer detection rate increased significantly. When a 6-core repeat biopsy was performed on this patient group, the prostate cancer detection rate decreased significantly. A high repeat biopsy core number in patients with high prostate volumes reduced the false-negative biopsy rate. It has been reported that the rate of prostate cancers in transitional zone biopsies is $1.8-8 \%(4,17)$. There is a transitional zone biopsy indication in patients with large prostates, no nodules, and high serum PSA (17). A study by Fernandes et al. included 58 patients diagnosed with prostate cancer who had had a 21-core repeat biopsy, and four of these $(6.9 \%)$ had cancer foci in the transitional zone (4). Especially in the case of patients with large prostates, to perform a 20-core repeat biopsy that includes the transitional zones, provides a positive contribution to the detection of prostate cancer. In our study, the rate of cancer detection increased as the core number of the repeat biopsy increased in the patient group with normal DRE ( $p=0.006)$. To perform more repeat core biopsies because of the spheric morphology of the prostate is logically an acceptable practice.

In the diagnosis of prostate cancer, borderline patients with PSA $<10$, PSAD $>0.15$ and prostate volume $>55 \mathrm{~mL}$ are in the most difficult subgroup. In these groups, a 20-core repeat biopsy is important for detecting hidden tumors. In our study, it has been determined that to perform a 20core repeat biopsy in this patient group significantly increases the rate of cancer detection.

Although the detection of ASAP in prostate biopsies is singly sufficient for the decision to perform a repeat biopsy, various parameters have been researched and nomograms developed in recent years, both to reduce the rate of redundant biopsies and increase the rate of positive prediction in cases where pathologies such as ASAP are present, as well as where these pathologies are not present. There are some studies that advocate using nomograms, emphasizing that nomograms obtain more accurate results than clinical markers. It is been suggested that a nomogram validated by Yanke et al. be used when deciding on a repeat biopsy (18). In this nomogram, parameters such as age, family history, PSA, PSA velocity, rectal exam findings, time elapsed following the initial biopsy procedure, the cumulative negative foci number, the presence of a diagnosis of HGPIN/ASAP in previous biopsies are used as risk factors (18). Nowadays, however, there is consensus about the performance of a repeat biopsy in cases where ASAP is detected. The focus of such research is on the repeat biopsy number, the biopsy regions, and the size of the biopsy sample. High cancer detection rates in regions different from the ASAP foci of the initial biopsy also support the performance of extended biopsies.

It has been reported that an increase in the number of biopsy cores does not cause a significant increase in the rate of complications $(19,20)$. A 20-core repeat biopsy can therefore be considered an appropriate method because the rate of 
prostate cancer detection is higher. This is important, particularly in the detection of significant disease (Gleason $\geq 7$ ) and in early diagnosis.

While the complication rate of minor or even prominent disorders was found to be similar in initial prostate biopsies and first repeat biopsies, morbidity in the third and fourth biopsies was found to be a little higher (21). Some patients may refuse a repeat biopsy due to either the risk of complications or because it is an invasive procedure. Therefore, it is important to increase the cancer detection rate by performing a high core number in the first repeat biopsy in order to reduce the number of repeat biopsies potentially required at a later stage. This application will also contribute to early cancer diagnosis.

\section{CONCLUSIONS}

In our study, in patients on whom a repeat biopsy was carried out because of ASAP, when the biopsy core number increased, the cancer detection rate increased significantly. When the foci where cancer was detected in a repeat biopsy were localized according to the ASAP foci in the initial biopsy, more than half of the cancer foci (54\%) were identified in contralateral biopsies. Performing 20-core repeat biopsies equally on all foci enhances the cancer detection rate significantly, especially in patients with a prostate volume $\geq$ $55 \mathrm{~mL}$. The practice of performing 20-core repeat biopsies equally on all foci may not only decrease the requirement for a second repeat biopsy, but also contribute to the early diagnosis of prostate cancer. However, this practice could increase the possibility of over-diagnosis and over-treatment.

\section{CONFLICT OF INTEREST}

None declared.

\section{REFERENCES}

1. Jemal A, Siegel R, Ward E, Murray T, Xu J, Smigal C, et al.: Cancer statistics, 2006. CA Cancer J Clin. 2006; 56: 106-30.

2. Patel U, Rickards $\mathrm{D}$ : Handbook of transrectal ultrasound\&biopsy of the prostate. Martin Dunitz Ltd, London. 2002; pp. 25-35.
3. Djavan B, Zlotta AR, Ekane S, Remzi M, Kramer G, Roumeguère $T$, et al.: Is one set of sextant biopsies enough to rule out prostate Cancer? Influence of transition and total prostate volumes on prostate cancer yield. Eur Urol. 2000; 38: $218-24$.

4. Campos-Fernandes JL, Bastien L, Nicolaiew N, Robert G, Terry S, Vacherot F, et al.: Prostate cancer detection rate in patients with repeated extended 21-sample needle biopsy. Eur Urol. 2009; 55: 600-6.

5. Epstein Jl, Potter SR: The pathological interpretation and significance of prostate needle biopsy findings: implications and current controversies. J Urol. 2001; 166: 402-10.

6. Olar A, Wheeler TM: Atypical Small Acinar Proliferation. In: J. Stephen Jones (ed.), Prostate Cancer Diagnosis. New York, Humana Press. 2013; pp. 255-68.

7. Moore CK, Karikehalli S, Nazeer T, Fisher HA, Kaufman RP Jr, Mian BM: Prognostic significance of high grade prostatic intraepithelial neoplasia and atypical small acinar proliferation in the contemporary era. J Urol. 2005; 173: $70-2$.

8. Hong YM, Lai FC, Chon CH, McNeal JE, Presti JC Jr.: Impact of prior biopsy scheme on pathologic features of cancers detected on repeat biopsies. Urol Oncol. 2004; 22: 7-10.

9. Zaytoun OM, Jones JS: Prostate cancer detection after a negative prostate biopsy: lessons learnt in the Cleveland Clinic experience. Int J Urol. 2011; 18: 557-68.

10. Punnen S, Nam RK: Indications and timing for prostate biopsy, diagnosis of early stage prostate cancer and its definitive treatment: a clinical conundrum in the PSA era. Surg Oncol. 2009; 18: 192-9.

11. Amin MM, Jeyaganth S, Fahmy N, Bégin L, Aronson S, Jacobson $S$, et al:: Subsequent prostate cancer detection in patients with prostatic intraepithelial neoplasia or atypical small acinar proliferation. Can Urol Assoc J. 2007; 1: 245-9.

12. Presti JC Jr: Prostate biopsy strategies. Nat Clin Pract Urol. 2007; 4: 505-11.

13. Ryu JH, Kim YB, Lee JK, Kim YJ, Jung TY: Predictive factors of prostate cancer at repeat biopsy in patients with an initial diagnosis of atypical small acinar proliferation of the prostate. Korean J Urol. 2010; 51: 752-6.

14. Allen EA, Kahane $\mathrm{H}$, Epstein JI: Repeat biopsy strategies for men with atypical diagnoses on initial prostate needle biopsy. Urology. 1998; 52: 803-7.

15. Chen ME, Troncoso P, Johnston D, Tang K, Babaian RJ: Prostate cancer detection: relationship to prostate size. Urology. 1999; 53: 764-8.

16. Letran JL, Meyer GE, Loberiza FR, Brawer MK: The effect of prostate volume on the yield of needle biopsy. J Urol. 1998; 160: 1718-21.

17. Liu IJ, Macy M, Lai YH, Terris MK: Critical evaluation of the current indications for transition zone biopsies. Urology. 2001; 57: 1117-20. 
18. Yanke BV, Gonen M, Scardino PT, Kattan MW: Validation of a nomogram for predicting positive repeat biopsy for prostate cancer. J Urol. 2005; 173: 421-4.

19. Zaytoun OM, Moussa AS, Gao T, Fareed K, Jones JS: Office based transrectal saturation biopsy improves prostate cancer detection compared to extended biopsy in the repeat biopsy population. J Urol. 2011; 186: 850-4.
20. Scattoni V, Maccagnano C, Zanni G, Angiolilli D, Raber $\mathrm{M}$, Roscigno M, et al.: Is extended and saturation biopsy necessary? Int J Urol. 2010; 17: 432-47.

21. Djavan B, Waldert M, Zlotta A, Dobronski P, Seitz C, Remzi $M$, et al.: Safety and morbidity of first and repeat transrectal ultrasound guided prostate needle biopsies: results of a prospective European prostate cancer detection study. J Urol. 2001; 166: 856-60.

Correspondence address: Erdogan Aglamis, MD Elazig Egitim ve Arastirma Hastanesi Uroloji Elazig, 23100, Turkey Telephone: + 9042 4238-1000 E-Mail: uroloji23@yahoo.com 